\title{
NEW EXPERIMENTAL STUDIES OF ICE GRAIN EJECTION BY MASSIVE GAS FLOW, IMPLICATIONS TO COMETS, ENCELADUS, TRITON AND MARS
}

\author{
A. Bar-Nun ${ }^{1}$, D. Laufer ${ }^{1}$, R. Jacovi ${ }^{1}$ and I. Pat-El ${ }^{1}$
}

\begin{abstract}
Ice grains are ejected whenever gases are flowing from gasladen amorphous ice upon its warming-up. We elucidated the mechanism of gas trapping when flowing onto a gas-free ice layer. The observations on ice grain ejection from comets, Enceladus, Triton and the Martian South Polar region are explained as well.
\end{abstract}

\section{Experimental procedures and results}

Two experimental setups were used to study ice grain ejection: (1) Thin ice samples were produced by flowing water vapor and gases, either mixed together or separately, on to a cold plate at $50 \mathrm{~K}$ (e.g. Bar-Nun et al. 1987; Laufer et al. 2013). In the case of $100 \mu \mathrm{m}$ layer of frozen $\mathrm{CO}_{2}$ covered by a $200 \mu \mathrm{m}$ thick layer of amorphous ice, upon uniformly warming up the sample, ice grains and jets of gas were measured by a quadrupole mass filter (MS), at a msec time resolution; (2) Few cm thick gas laden amorphous ice layers were formed in a one of its kind machine (Bar-Nun et al. 2003). Alternatively, in this machine separate cm thick layers of frozen gas and water ice could have been deposited, one on the top of the other.

The new experimental studies on thin (up to $200 \mu \mathrm{m}$ ) ice layers confirmed our previous findings (Bar-Nun et al. 1987) on ice grain ejection during the annealing of gas-laden amorphous ice and during its transformation into cubic ice. A massive gas flow, from the interior, breaks the ice structure and is imbedded in the amorphous ice, to be released from it during its annealing and transformation to cubic ice as seen in Figure 1.

A new millisecond time resolution revealed a huge flux of tiny ice particles emanating from the ice (Fig. 2).

1 Department of Geophysical Atmospheric and Planetary Sciences, Tel-Aviv University, Tel-Aviv 69978, Israel 


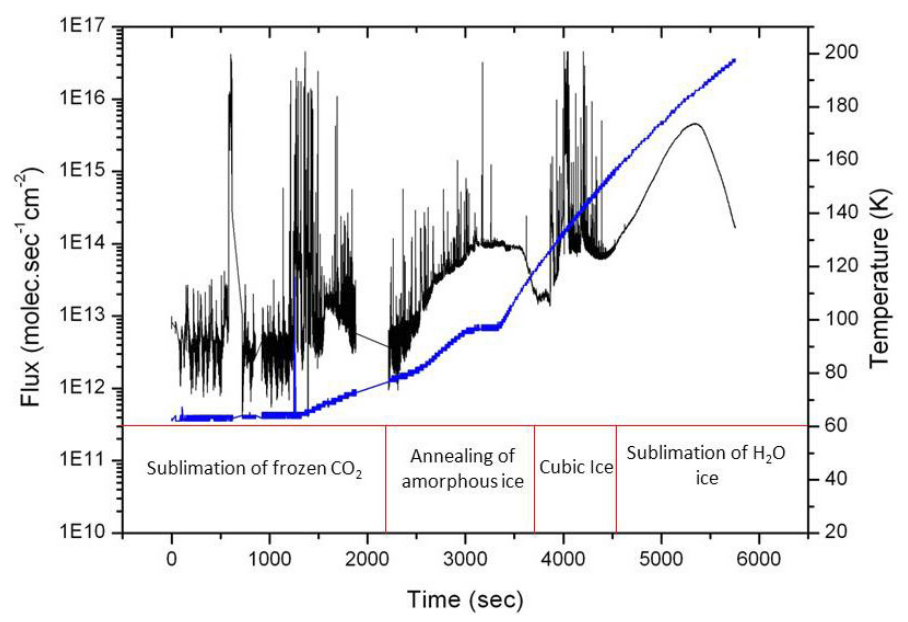

Fig. 1. MS record of ice grain ejection during the flow of $\mathrm{CO}_{2}$ through an overlying $200 \mu \mathrm{m}$ ice layer.
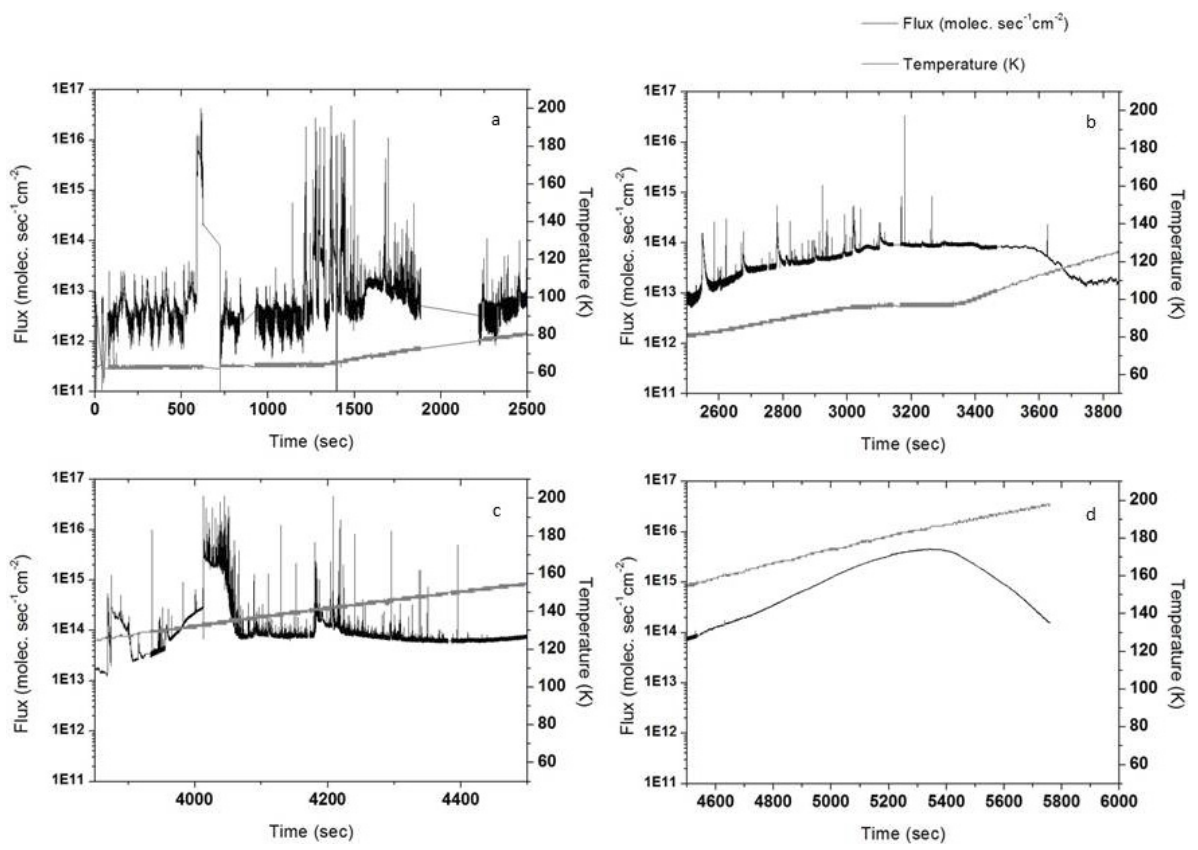

Fig. 2. MS analysis of the ejected ice grains.

Using our measured density of $250 \mathrm{~kg} \mathrm{~m}^{-3}$ (Laufer et al. 2005), the size distribution of the ice grains ranges from $1.4 \mu \mathrm{m}$ up to $145 \mu \mathrm{m}$, with the dominance of the smaller ones, as observed in comets (Fig. 3). 


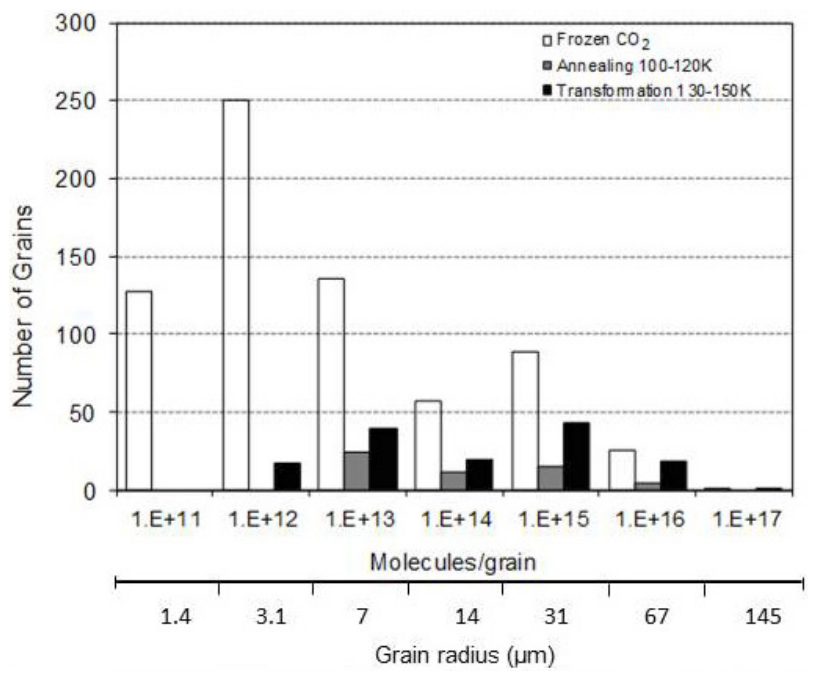

Fig. 3. Size distributions of the ice grains in the different temperature ranges, measured for all grains recorded in Figure 2.

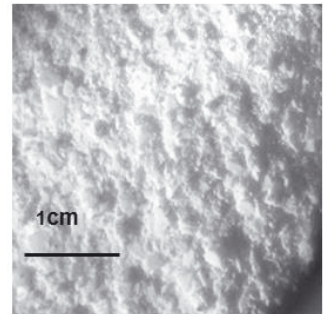

$1 \mathrm{~min}$

a

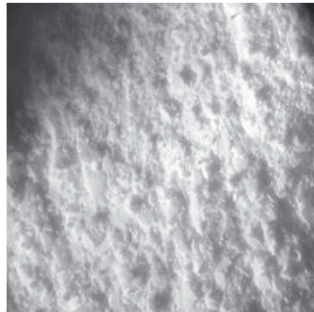

$40 \mathrm{~min}$

b

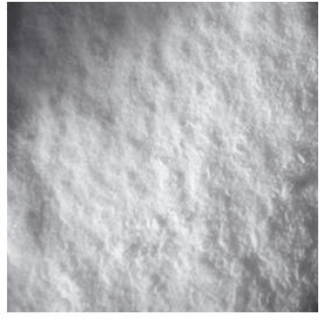

$65 \mathrm{~min}$

Fig. 4. Coverage of the surface, by ejected ice grains of a large ice sample.

In large [20 cm diameter and a few $\mathrm{cm}$ thick] samples of gas-laden amorphous ice, a huge flux of ejected ice grains covers over the time the ice surface, as shown experimentally (Fig. 4).

Gas jets accompanying ice grain ejection, as observed in Comets, Enceladus, Triton and Mars South Pole in spring can be explained by our experimental findings:

1. On comets and Enceladus, massive gas flow breaks the overlying ice layer and propels the ice debris. It also carries ice grains from the channels through which gas flows. The measured speed of ice grains $1.5 \mathrm{~m} \mathrm{~s}^{-1}$, reaches speeds of $60 \mathrm{~m} \mathrm{~s}^{-1}$ (Porco et al. 2006) due to Enceladus' lower gravity. 
2. On Triton, Plumes of ice and dark material are ejected up to $8 \mathrm{~km}$ and are carried by the wind, to form dark streaks on the surface. Given Triton's gravity the gas jets require a higher speed against the gravity, therefore, the source of the outflowing gas jets is in deeper layers.

3. On Mars during springtime in the South Pole, streaks of dark material are formed by jets of gas carrying dark dust grains and carried down wind. Here our experiments show that jets of $\mathrm{CO}_{2}$ cannot be formed by mere sublimation of frozen $\mathrm{CO}_{2}$. Rather a mixture of frozen $\mathrm{CO}_{2}$ and $\mathrm{H}_{2} \mathrm{O}$ is required to form these jets.

This research was supported by a BSF grant 2006339 and by the Ministry of Science and Technology grant 3-8349.

\section{References}

Bar-Nun, A., Dror, J., Kochavi, E., \& Laufer, D., 1987, Phys. Rev. B, 35, 2427

Bar-Nun, \& Laufer, D., 2003, Icarus, 178, 248

Laufer, D., Pat-El, I., \& Bar-Nun, A., 2005, Icarus, 178, 248

Laufer, D., Bar-Nun, A., Pat-El, I., \& Jacovi, R., 2013, Icarus, in press

Porco, C.C., et al., 2006, Science, 311, 1393 